Portland State University

PDXScholar

\title{
Tinnitus, Depression, Anxiety, and Suicide in Recent Veterans: A Retrospective Analysis
}

\author{
Erin Martz \\ Veterans Affairs Portland Healthcare System \\ Chennettee Jelleberg \\ Portland State University \\ Deborah D. Dougherty \\ Intellica Corporation \\ Charles Wolters \\ Intellica Corporation \\ Aaron Schneiderman \\ Veterans Health Administration, District of Columbia.
}

Follow this and additional works at: https://pdxscholar.library.pdx.edu/coun_fac

Part of the Counselor Education Commons

Let us know how access to this document benefits you.

Citation Details

Martz, E., Jelleberg, C., Dougherty, D. D., Wolters, C., \& Schneiderman, A. (2018). Tinnitus, depression, anxiety, and suicide in recent veterans: a retrospective analysis. Ear and hearing, 39(6), 1046-1056.

This Article is brought to you for free and open access. It has been accepted for inclusion in Counselor Education Faculty Publications and Presentations by an authorized administrator of PDXScholar. Please contact us if we can make this document more accessible: pdxscholar@pdx.edu. 


\title{
Tinnitus, Depression, Anxiety, and Suicide in Recent Veterans: A Retrospective Analysis
}

\author{
Erin Martz ${ }^{1}$, Chennettée Jelleberg ${ }^{2}$, Deborah D. Dougherty ${ }^{3}$, Charles Wolters ${ }^{3}$, \\ Aaron Schneiderman ${ }^{4}$
}

Objectives: The purpose of this project was to investigate whether there is an association between tinnitus diagnosis and suicide and whether depression and anxiety strengthen that association. Given that tinnitus is the top service-connected disability among U.S. Veterans (Veterans Benefits Administration, 2016) and that suicide among Veterans has been occurring at a higher frequency as compared with community suicide rates (Hoffmire et al., 2015), the possible associations between tinnitus and suicide will be explored. Co-occurring physical conditions also will be examined to determine if they increase the risk of suicide in the context of tinnitus.

Design: Administrative health care data related to Operations Enduring Freedom/Iraqi Freedom/New Dawn (OEF/OIF/OND) were used to identify Veterans who accessed the Veterans Administration (VA) health care system from January 1, 2002, to December 31, 2011. Veterans who were deceased as of December 2011 were identified using the National Death Index (NDI) files. Tinnitus cases were followed until either they were deceased or to the end of the study period. The International Classification of Diseases 9th Revision Clinical Modification (ICD-9-CM) codes were used to identify all conditions and diseases. As per mortality research standards, International Classification of Diseases 10th Revision (ICD-10) codes were used to identify cause of death.

Results: Of 769,934 OEF/OIF/OND Veterans receiving VA care January 2002 to December 2011, 15\% ( $n=116,358)$ were diagnosed with tinnitus. Of these Veterans diagnosed with tinnitus, $21 \%$ were also diagnosed with depression, another $8 \%$ with anxiety, and another $17 \%$ with both depression and anxiety. Fifty-four percentage were identified as having tinnitus without depression or anxiety. Among individuals with tinnitus, $41.9 \%$ had co-occurring hearing loss. Suicide rates were lower among Veterans with tinnitus than Veterans without tinnitus. Co-occurring diagnoses of mentalhealth conditions did not significantly increase the risk of suicide.

Conclusions: The study results do not confirm clinical and anecdotal reports that tinnitus could be related to suicide among Veterans. However, tenets from rehabilitation psychology suggest that the onset of chronic impairment or disability does not predict an individual's subsequent psychological states; other personal attributes may be more influential. Health care professionals, such as audiologists and psychologists, should be cognizant of the associations between tinnitus and mental health issues and be prepared to address the psychological needs of individuals who have tinnitus.

Key words: Anxiety, Depression, Suicide, Tinnitus, Veterans.

(Ear \& Hearing 2018;39;1046-1056)

\section{INTRODUCTION}

Tinnitus (ringing in the ears) is the most prevalent serviceconnected disability in the Veterans Administration (VA)

${ }^{1}$ Rehability Oregon, and the National Center of Rehabilitative Auditory Research, Veterans Affairs Portland Healthcare System, Portland, Oregon; ${ }^{2}$ Portland State University, Portland, Oregon; ${ }^{3}$ Intellica Corporation, San Antonio, Texas; and ${ }^{4}$ Veterans Health Administration, Office of Patient Care Services, Post Deployment Health Services, Epidemiology Program, Washington, District of Columbia.

Supplemental digital content is available for this article. Direct URL citations appear in the printed text and are provided in the HTML and text of this article on the journal's Web site (www.ear-hearing.com). system (Veterans Benefits Administration, 2016). As of August 3, 2015, among 224,610 Veterans registered in the Million Veteran Program, the prevalence of tinnitus was 32.3\% (Gaziano et al., 2016). Although previous studies have explored whether tinnitus may be connected to suicide (Jacobson \& McCaslin, 2001; Lewis et al., 1994; Pridmore et al., 2012), none of them specifically addressed Veterans with tinnitus.

Recent research on suicide rates among Veterans suggested that suicide occurs more frequently among Veterans than nonVeterans (Hoffmire et al., 2015), and that in 2014, an average of 20 Veterans committed suicide every day (Veterans Affairs, 2016). Veterans aged 18-29 years had the highest rate of suicide in 2014; approximately 33 per 100,000 among females and 87 per 100,000 among males. The lowest rates were seen among males aged 70-79 (20 per 100,000) and females aged 70 or more (4 per 100,000) (Veterans Affairs, 2016). Kemp and Bossarte (2012) assessed U.S. Veteran suicide rates from 2009 to 2012 by examining data reported in 21 states, finding that $18.3 \%$ of the total number of suicide cases $(\mathrm{N}=147,763)$ had a history of U.S. military service listed on their death certificates. Research comparing U.S. Veterans deployed to Iraq or Afghanistan and non-deployed Veterans indicated that deployment to a war zone itself was not a risk factor for suicide (Kang et al., 2015; Reger et al., 2015).

Yet, tinnitus can be an occupational hazard for military members serving in a war zone. One blast injury can cause tinnitus (Cave et al., 2007; Yankaskas, 2013); in addition to other injuries, hearing loss, auditory and cognitive processing impairments (Fausti et al., 2009; Myers et al., 2009). While some researchers (Hoare et al., 2014) state that there is a $90 \%$ cooccurrence of tinnitus with some degree of hearing loss, recent empirical research varies widely on the reported co-occurrence of tinnitus and hearing loss, from 3\% (Table 1, Joo et al., 2015) to $56 \%$ (Kochkin et al., 2011). A traumatic brain injury (TBI) during military deployment may result in concomitant tinnitus (Cave et al., 2007; Yurgil et al., 2016). Predeployment TBI among service members without predeployment tinnitus significantly increased the risk of postdeployment tinnitus among military members (Yurgil et al., 2016).

Previous studies on tinnitus and suicide (Jacobson \& McCaslin, 2001; Lewis et al., 1994; Pridmore et al., 2012) were limited by their reliance on case studies, small sample sizes, or use of community samples, none of which identified Veterans with tinnitus. This study explores associations between tinnitus and suicide among Veterans diagnosed with tinnitus, while considering the moderation of this association by depression and anxiety. Given that depression and anxiety have been linked to suicide in numerous research studies (Chesney et al., 2014; Kanwar et al., 2013; Turecki \& Brent, 2016), those two variables were examined in the present study to investigate whether they were associated with suicide. Co-occurring physical conditions 
TABLE 1. Characteristics of OEF/OIF/OND Veterans with a Diagnosis of Tinnitus, Anxiety, and Depression, January 2002 to December 2011

\begin{tabular}{|c|c|c|c|c|c|c|}
\hline Characteristics & $\begin{array}{l}\text { Veterans Who } \\
\text { Received VA Health } \\
\text { Care 2002-2011 }\end{array}$ & $\begin{array}{l}\text { Veterans Receiving } \\
\text { Health Care and } \\
\text { Diagnosed with } \\
\text { Tinnitus }\end{array}$ & \multicolumn{4}{|c|}{ Subgroups of Those Diagnosed With Tinnitus } \\
\hline Study population & 769,934 & $116,358(15.1)$ & $9,301(8.0)$ & $24,524(21.1)$ & $19,556(16.8)$ & $62,976(54.1)$ \\
\hline \multicolumn{7}{|l|}{ Demographic characteristics } \\
\hline \multicolumn{7}{|l|}{ Sex } \\
\hline \multicolumn{7}{|l|}{ Age at first visit (y) } \\
\hline 25 or younger & $245,594(31.9)$ & $35,895(30.8)$ & $3,550(38.1)$ & $7,739(31.6)$ & $6,512(33.3)$ & $18,094(28.7)$ \\
\hline $26-30$ & $187,710(24.4)$ & $25,424(21.9)$ & $2,299(24.7)$ & $5,720(23.3)$ & $4,574(23.4)$ & $12,831(20.4)$ \\
\hline $31-40$ & $167,409(21.7)$ & $24,785(21.3)$ & $1,779(19.1)$ & $5,514(22.5)$ & $4,569(23.4)$ & $12,923(20.5)$ \\
\hline $41-50$ & $135,441(17.6)$ & $23,108(19.9)$ & $1,314(14.1)$ & $4,191(17.1)$ & $3,070(15.7)$ & $14,533(23.1)$ \\
\hline 51 or older & $33,777(4.4)$ & $7,145(6.1)$ & 359 (3.9) & $1,360(5.6)$ & $831(4.3)$ & $4,595(7.3)$ \\
\hline Other/unknown & $220,598(28.7)$ & $32,665(28.0)$ & $2,224(23.8)$ & 6,503 (26.4) & $5,155(26.4)$ & $18,783(29.7)$ \\
\hline \multicolumn{7}{|l|}{ Branch } \\
\hline Air Force & $95,902(12.5)$ & $13,586(11.7)$ & $700(7.5)$ & $1,621(6.6)$ & $1,372(7.0)$ & $9,893(15.7)$ \\
\hline Army & $466,269(60.6)$ & $70,494(60.6)$ & $5,980(64.3)$ & $16,844(68.7)$ & $13,675(69.9)$ & $33,995(54.0)$ \\
\hline Coast Guard & $890(0.1)$ & $103(0.1)$ & $8(0.1)$ & $10(0.0)$ & $15(0.1)$ & $70(0.1)$ \\
\hline Marine Corps & $105,247(13.7)$ & 20,667 (17.8) & $1,921(20.7)$ & $4,116(16.8)$ & $3,014(15.4)$ & $11,616(18.4)$ \\
\hline Navy & $101,626(13.2)$ & $11,508(9.9)$ & $692(7.4)$ & $1,933(7.9)$ & $1,480(7.6)$ & $7,403(11.8)$ \\
\hline \multicolumn{7}{|l|}{ Rank } \\
\hline Enlisted & 701,792 (98.9) & $106,702(91.7)$ & $8,823(94.9)$ & 23,362 (95.3) & $18,756(95.9)$ & $55,761(88.5)$ \\
\hline Officer/Warrant Officer & $68,142(1.1)$ & $9,656(8.3)$ & $478(5.1)$ & $1,162(4.7)$ & $800(4.1)$ & $7,216(11.5)$ \\
\hline \multicolumn{7}{|l|}{ Component } \\
\hline
\end{tabular}

Percentage values are shown in parentheses.

were also analyzed to determine if they increased the risk of suicide among Veterans with tinnitus.

\section{Empirical Research and Case Studies on Tinnitus and Suicide}

Lewis et al. (1992) published six case studies that focused on individuals who had died in violent circumstances and had tinnitus. They noted that all six individuals had reported experiencing depression, but stated that the associations among tinnitus, depression, and suicide were unclear. The researchers suggested that depression should be studied as a risk factor, and that a larger number of cases would be needed for an analysis of those associations. Lewis et al. (1994) circulated a 20-item Tinnitus Suicide Questionnaire to audiology clinics worldwide; 50 clinics responded with data. The data indicated that there were 28 cases of suicide among individuals reporting tinnitus. Notably, 20 of the 28 cases were males and 10 individuals had a history of mental-health issues before the diagnosis of tinnitus.
In a subset of 23 cases that included relevant information, nine individuals had committed suicide within 1 year of the onset of tinnitus and an additional two individuals within the second year (i.e., totaling almost half the sample by the second year after tinnitus onset). The authors suggested that these data reflect that the onset of tinnitus is "a significant life event" (p. 53).

Jacobson and McCaslin (2001) conducted a literature review on the topic of tinnitus and suicide during the time-frame of 1966-2001. Of the 12 articles found, the researchers excluded eight that dealt with tinnitus triggered by self-poisoning. Of the four remaining articles, the sample sizes were small, ranging from 1 to 28 individuals. The authors asserted that their review of these four articles did not suggest a predictive relationship between tinnitus and suicide. They stated that "it is not tinnitus per se that results in suicide, but concomitant psychiatric conditions that amplify the effect of tinnitus on the individual patient" (Jacobson \& McCaslin, 2001, p. 495).

Lewis (2002) wrote a letter to the editor about the results and interpretation of his two published case-study reports with 
data on suicide and tinnitus. He noted that "people suffering with depression are less resilient and able to cope with their tinnitus, but this cannot be the only explanation for the possible high incidence of suicide in a tinnitus population" (p. 339). In a response, Jacobson and McCaslin (2001) asserted that a population-based study was needed to examine depression-related suicide, or whether tinnitus was the main factor driving individuals to suicide. Both letters suggested that suicide among individuals with tinnitus was a complex issue that needed further study of the interaction between tinnitus and mental-health variables.

Pridmore et al. (2012) examined the possible association between suicide and tinnitus by searching public records via newspapers and conducting an internet search for stories within the last decade. They provided short descriptions of four cases in which suicide appeared to be related to tinnitus. The researchers acknowledged that due to the nature of the data presented in their article, the results could not provide arguments for or against a causal relationship between tinnitus and suicide. They did suggest that health professionals should be compassionate toward individuals who are bothered by their tinnitus and make sure to refer those who are significantly distressed about their tinnitus to mental-health providers.

Seo et al. (2016) conducted one of the first large research studies that investigated the association between suicidal behavior and tinnitus (i.e., both suicidal ideation and suicide attempts, but not completed suicide). They examined data collected from 17,446 Koreans in the Korean National Health and Nutrition Examination Survey. The existence of tinnitus was assessed by asking "Within the past year, did you ever hear a sound (buzzing, hissing, ringing, humming, roaring, machinery noise) originating in your ear?" Severity level was determined by asking "How severe is this noise in your daily life?" Suicidal ideation was assessed by asking "In the last 12 months, did you think about committing suicide?" If a subject responded yes to that question, they were asked about any suicide attempts. A chisquare analysis indicated a significant difference among suicidal ideation and suicidal attempts related to tinnitus existence: $20.9 \%$ of individuals with tinnitus reported suicidal ideation, compared with $12.2 \%$ of individuals without tinnitus, while $1.2 \%$ of individuals with tinnitus reported suicide attempts, compared with $0.6 \%$ of individuals without tinnitus.

\section{Associations Between Tinnitus, Depression, and Anxiety}

For decades, research has confirmed an association between depression and tinnitus. In one study, Harrop-Griffiths et al. (1987) conducted a hospital-based, case-control study to examine associations between psychiatric diagnoses among 21 individuals with severe tinnitus and 14 individuals with hearing loss. They found that individuals with tinnitus had significantly higher prevalence rates of lifetime and current major depression than the group with hearing loss. Sullivan et al. (1988) found that a group of 40 individuals with tinnitus had a significantly higher number of current and lifetime diagnoses of major depression compared with a control group of 14 individuals from the otolaryngological clinic who had a hearing impairment. They suggested that "tinnitus becomes disabling if and when it becomes associated with depression" (p. 289).

Sullivan et al. (1993) noted that their research using nortriptyline for tinnitus-related disability suggested that "the relationship between tinnitus and depression may be bi-directional rather than uni-directional. Tinnitus (like chronic pain) may prompt a depressive episode that may impede habituation to the aversive sensation and thereby increase the severity of the tinnitus experienced" (p. 2258). Erlandsson and Hallberg (2000) examined 13 predictors of tinnitus-related quality of life among 122 cases. Three psychological predictors (i.e., impaired concentration, depression, and interpersonally related emotional problems) explained $61 \%$ of the variance in quality of life. Jacobson and McCaslin (2001) noted from their review of the literature that depression was the primary psychiatric issue among individuals who had tinnitus and had committed suicide; they suggested that instead of focusing on the possibility of tinnitus as a cause of suicide, "it is more appropriate to state that 'the practicing audiologist and otologist need to be aware of the possible risk of suicide among clinically depressed tinnitus sufferers"' (p. 495).

Several studies have noted a link between tinnitus and anxiety. Marciano et al. (2003) studied 75 individuals with tinnitus and co-occurring psychiatric issues and found that 22 of these individuals met Diagnostic and Statistical Manual of Mental Disorders, 4th Edition, criteria for an anxiety disorder, while an additional 20 individuals had a diagnosis of an affective disorder (that may include depression). Bartels et al. (2008) surveyed 265 individuals with tinnitus who were seen within a hospital otolaryngology department. Individuals were categorized into one of four groups: (1) 27 individuals in the anxiety-only group; (2) 26 individuals in the depression-only group; (3) 104 in the anxietyplus-depression group; and (4) 108 in the no-symptom group. When compared with the group with no symptoms, those reporting depression, anxiety, or depression and anxiety symptoms showed significant differences on this study's four outcomes measuring general distress, tinnitus-related distress, tinnitus coping, and quality of life. The researchers concluded that cooccurring depression and anxiety have an "additive effect" in worsening multiple mental-health and quality of life variables.

A Norwegian study by Krog et al. (2010) examined tinnitus and depression, anxiety, self-esteem, and subjective well-being from 1995 to 1997 among 51,574 individuals with hearing loss. The group was divided into four "symptom level" (i.e., severity) categories: low, intermediate, high, or no tinnitus. Researchers found that individuals with tinnitus at any severity level had significantly higher scores on anxiety and depression than individuals without tinnitus, after adjusting for age. Yet, small effects were found for tinnitus symptoms on anxiety and depression levels.

Nondahl et al. (2011) examined epidemiological data from the Beaver Dam Offspring Study, focusing on hearing impairments and a range of outcomes between 2005 and 2008 . Multivariate analysis indicated that tinnitus was significantly associated with depressive symptoms. Langguth et al. (2011) investigated the idea of a complex association between tinnitus and depression, exploring the idea that a causal relationship between tinnitus and depression should not be assumed, because the reverse association may occur: depression could trigger an exacerbation of tinnitus. They suggested that neurobiological factors may influence whether tinnitus affects individuals' emotional states after tinnitus onset. They pointed out that multiple symptoms related to tinnitus (e.g., insomnia, irritability, concentration problems, fatigue, social withdrawal) were similar to symptoms of depression, which may confound the understanding of psychological reactions to tinnitus. They suggested that the reported symptoms may not necessarily represent a 
diagnosis of depression, but rather symptoms of more general psychological distress.

In a large study on hearing $(\mathrm{N}=171,728)$, McCormack et al. (2015) found associations between tinnitus severity, anxiety, and depression symptoms: individuals with severe tinnitus had an increased likelihood of symptoms of depression (odds ratio $=1.27$ ) and an increased likelihood of more anxiety symptoms (odds ratio $=1.11$ ) than those without tinnitus. The authors suggested that "high depression and/or anxiety symptoms limit a person's ability to cope with tinnitus and thus they perceive it as more bothersome compared to people with low level of depression or anxiety" (McCormack et al., 2015, p. 603). They cautioned that the results indicate an association, not causation.

A population-based study (Bhatt et al., 2017) examined tinnitus data gathered by the 2007 National Health Interview Series; 21.4 million individuals reported experiencing tinnitus, while $26.1 \%$ of that group reported having problems with depression and anxiety. Compared with individuals without tinnitus, those with tinnitus were significantly more likely to report depression and anxiety. The aforementioned research studies suggest that depression and anxiety are commonly experienced among individuals with tinnitus, and thus, the association between tinnitus and suicide may be modified by these two psychological factors.

While the following Veteran studies did not focus on tinnitus, their research findings were informative for the present study. Dobscha et al. (2014) conducted a case-control study to examine the risk factors for suicide among Veterans receiving VA health care. They found that depression, anxiety disorder, functional decline, and suicidal ideation were associated with a greater risk of suicide. Zivin et al. (2015) examined data on mortality associated with depression among Veterans and found that co-occurring physical (e.g., heart disease, respiratory illness, cerebrovascular disease, diabetes, nephritis, influenza, Alzheimer's disease, septicemia, Parkinson's disease, and hypertension) and psychiatric conditions increased the risk of mortality, the exception being anxiety and posttraumatic stress disorder, which decreased the risk of mortality. Finally, a systematic review of 19 studies on the risk factors for suicide (Hawton et al., 2013) indicated that a diagnosis of depression was the most common psychiatric condition among individuals who committed suicide; anxiety was also reported as a risk factor.

\section{Hypotheses}

The following study hypotheses were developed as a result of the literature review:

1. A diagnosis of tinnitus is positively associated with suicide.

2. Having a diagnosis of depression or anxiety will strengthen the association between a tinnitus diagnosis and suicide.

\section{MATERIALS AND METHODS}

\section{Ethics}

Before conducting this research, permission was sought from the VA Portland Healthcare System's Research and Development department and the Institutional Review Board (IRB). It was decided that the present study's secondary data analysis was a "science-only study" and thus was exempt from IRB approval. The Research and Development department approved this study's procedures.

\section{Data Sources}

To form the study population, a roster file of Operations Enduring Freedom/Iraqi Freedom/New Dawn (OEF/OIF/OND) Veterans was obtained from the DOD's Defense Manpower Data Center (DMDC). This dataset contained demographic data (i.e., date of birth, sex, and race) and service-related information [i.e., rank, component (Active Duty, Reserve/Guard), and military branch]. The roster file was refined to include only OEF/OIF/OND Veterans who separated from service between January 1, 2002, and December 31, 2011, and who were not killed in action.

National Death Index (NDI) files produced by the National Center for Health Statistics (NCHS) were used to identify $\mathrm{OEF} / \mathrm{OIF} / \mathrm{OND}$ Veterans who died after separating from the military. The mortality file constructed for VA use contains vital status (alive, deceased), and if deceased, the date of death and the International Classification of Diseases, version 10 (ICD-10) cause of death. This file was restricted to include only OEF/OIF/OND Veterans who died on or before December 31, 2011, the most recent information available from NCHS. The 28 major death categories developed by the National Injury and Occupational Safety and Health Agency were regrouped into three categories. They included: (1) suicide, a subset of the major cause-of-death category "Violence," was placed in its own category; (2) unintentional injury, which included violence (assault, homicide, and terrorism) and accidents (vehicular and falls); and (3) healthrelated causes of death.

A data file of OEF/OIF/OND Veterans having health care encounters between January 1, 2002, and December 31, 2011, was constructed using the inpatient and outpatient encounter records from the Department of Veteran Affairs National Patient Care Database (NPCD). These records contained the International Classification of Disease, Ninth Revision, Clinical Modification (ICD-9-CM) codes used to create the medical condition variables. This dataset was compared with the OEF/ OIF/OND roster file; only Veterans matching to the inpatient/ outpatient encounter dataset were included in the analysis.

Three groups of Veterans were excluded from the study, those who: (a) were found in the NDI mortality file and had a date of death that was earlier than his or her separation date; (b) were found in the DMDC file and had death as a reason for separation from service; and (c) had outpatient/inpatient encounters in the NPCD, that all occurred after their dates of death.

\section{Data Analysis}

Data analysis incorporated univariate statistics (i.e., frequency tables) to examine demographic and service characteristics of the study sample. Chi-square analyses identified unadjusted relationships comparing two categorical variables of interest, such as comparing the proportion in each tinnitus subgroup between branches of service. Cause of mortality (all cause, suicide, unintentional injury, and health-related) was the dependent variable in separate logistic regression models. The independent variables in each model included presence of a tinnitus diagnosis, attempted self-harm encounters, audiology or mental-health clinic visits, co-occurring health conditions, and age at first health encounter. The statistical analyses were accomplished using Statistical Analysis Software (SAS), Version 9.3. 


\section{Creation of the Tinnitus and Mental-Health Condition Variables}

All diagnostic fields (13 for inpatient records and 10 for outpatient records) on the health encounter records were used to identify tinnitus cases. The presence or absence of unspecified depressive disorders $(296.2 \times, 296.3 \times, 296.9 \times, 311)$ or anxiety states, $(300.00)$ was determined for the tinnitus cases. To get a full understanding of the relationships between tinnitus, depression, and anxiety, the following tinnitus subgroups were created: (a) tinnitus and depression, no anxiety; (b) tinnitus and anxiety, no depression; (c) tinnitus with depression and anxiety; (d) tinnitus-only; and (e) no tinnitus (see Appendix A, Supplemental Digital Content 1, http:// links.lww.com/EANDH/A417, for the ICD-9-CM codes used to define each category). All tinnitus cases were followed until they were either deceased or to the end of the study period.

After reviewing the initial results, attempted self-harm encounters (ICD9 = E950-E958) were added to the analysis to better explain the outcomes. To examine the associations between suicide and VA health care services received for tinnitus, the receipt of audiology or mental health services were added to the regression models, identified by clinic stop-codes in the outpatient encounter records (see Appendix A, Supplemental Digital Content 1, http://links.lww.com/EANDH/A417).

\section{Person-Year Estimates and Crude Mortality Rates}

To produce the person-year estimates for the computation of mortality rates, all Veterans having a separation date between January 1, 2002, and December 31, 2011, were placed in the person-year estimation group. The person-year estimate for a Veteran who was not deceased was the difference between the date of initial VA health care visit and December 31, 2011, the study endpoint, divided by 365 . The person-year estimate for a deceased Veteran was the difference between the initial VA health care visit and the date of death divided by 365 . The rates presented in Table 3 are crude rates; for each table cell, the crude rate is the number of cause-specific deaths divided by the person-years per 100,000 Veterans within the specified Veteran group. To facilitate the assessment of mortality among Veterans with tinnitus, mortality rates (Table 3 ) were calculated on three levels: a) Level 1: all who received healthcare; b) Level 2: all diagnosed with tinnitus; and c) Level 3: tinnitus subgroups.

\section{RESULTS}

\section{Demographic and Service Characteristics}

Of the 769,934 OEF/OIF/OND Veterans receiving VA healthcare January 2002 to December 2011, 15\% $(n=116,358)$ were diagnosed with tinnitus (see Table 1). Among those diagnosed with tinnitus, the proportion of suicide deaths $(20.4 \%)$ was lower than unintentional injury (45.6\%) or health-related (33.9\%) deaths.

When further aggregated by anxiety and depression, $21 \%$ of OEF/OIF/OND Veterans diagnosed with tinnitus were also diagnosed with depression, another $8 \%$ with anxiety, and another $17 \%$ with both depression and anxiety. Fifty-four percentage were identified as having tinnitus-only. Approximately $5 \%$ of both the tinnitus-only and tinnitus with anxiety subgroups and $8-9 \%$ of both the tinnitus with depression and depression/anxiety subgroups were female. Forty-nine percentage of the tinnitusonly subgroup was age 30 or less, compared with $55-57 \%$ of the tinnitus with depression and with depression/anxiety groups and $63 \%$ of the tinnitus with anxiety group. The tinnitus-only subgroup contained a lower proportion of Army Veterans (54\%) compared with the proportion within each of the other subgroups (between $61 \%$ and $70 \%$ ). A chi-square test for independence was used to determine if Veterans in the tinnitus groups were equally distributed across the categories of each of the demographic and service characteristics. All tests were significant at the $p<0.05$ level, indicating that there is a relationship between tinnitus group and demographic/service characteristic categories. The fact that the data were not equally distributed across the independent variable categories could be a result of the mental-health conditions themselves, not the combined effect of tinnitus and mental health. The subsequent statistical analysis addressed this theory.

\section{Health Encounter Characteristics and Co-Occurring Conditions}

Sixteen physical conditions were identified by a research review as commonly co-occurring with tinnitus. In the present sample, among Veterans diagnosed with tinnitus, $65 \%$ were diagnosed with a co-occurring physical condition. The most common were hearing loss $(41.9 \%)$, TBI $(22.9 \%)$, and blood-vessel disorders, such as hypertension or atherosclerosis $(19.3 \%)$. Almost $1 \%$ of individuals with tinnitus had an attempted self-harm encounter, while over $92 \%$ had an audiology or mental-health clinic stop.

Among the tinnitus subgroups, Veterans diagnosed with tinnitus-only did not utilize the VA health care system as much as those Veterans in the other three subgroups. Veterans in the tinnitus-only subgroup had an average of 25.6 VA health care encounters $($ median $=13)$ compared with the other subgroups who had an average of 55-127 encounters (median $=28-83$; Table 2). These utilization differences may be due to the need for additional mental-health services among those with a cooccurring mental-health condition.

Co-occurring physical conditions were present among $74 \%$ of Veterans diagnosed with tinnitus/depression and $77.2 \%$ of Veterans diagnosed with tinnitus/depression/anxiety. Approximately $44 \%$ of Veterans in the tinnitus/depression and tinnitus/ depression/anxiety subgroups were also diagnosed with hearing loss, compared with approximately $40 \%$ in the tinnitus/anxiety and tinnitus-only subgroups.

\section{Mortality Rates}

There were 160.1 deaths per 100,000 person-years among all Veterans who received health care between January 2002 and December 2011 (Table 3, level 1). The mortality rate was 131.6 deaths per 100,000 person-years when assessing all Veterans diagnosed with tinnitus (level 2). Within this group, other unintentional injury deaths accounted for the highest death rate (60.0 per 100,000 person-years), followed by health-related deaths (44.7) and suicide (26.9 per 100,000 person-years).

Looking at the third level of mortality rates, that is, those within the tinnitus subgroups, a higher rate of death was found among Veterans diagnosed with tinnitus and depression and/or anxiety than those with tinnitus-only. Specifically, there were 179.2 deaths (i.e., all-cause mortality) per 100,000 person-years among Veterans in the tinnitus/anxiety/depression group compared with 97.3 deaths per 100,000 person-years among Veterans diagnosed with tinnitus-only. The rate of suicide was 24.5 per 100,00 in the tinnitus/anxiety group, 39.5 per 100,000 in the 
TABLE 2. Encounter Characteristics and Co-occurring Conditions among OEF/OIF/OND Veterans With a Diagnosis of Tinnitus, Anxiety, and Depression, January 2002 to December 2011

\begin{tabular}{|c|c|c|c|c|c|}
\hline & $\begin{array}{l}\text { Veterans Receiving } \\
\text { Health Care and } \\
\text { Diagnosed With } \\
\text { Tinnitus }\end{array}$ & \multicolumn{4}{|c|}{ Subgroups of Those Diagnosed With Tinnitus } \\
\hline Study population & 116,358 & $9,301(8.0)$ & $24,524(21.1)$ & $19,556(16.8)$ & $62,976(54.1)$ \\
\hline \multicolumn{6}{|c|}{ Encounter characteristics } \\
\hline \multicolumn{6}{|c|}{ Attempted self-harm encounter } \\
\hline \multicolumn{6}{|c|}{ Audiology or mental health clinic stop } \\
\hline Yes & $107,390(92.3)$ & $8,848(95.1)$ & $23,869(97.3)$ & $19,335(98.9)$ & $55,338(87.9)$ \\
\hline No & $8,968(7.7)$ & $453(4.9)$ & $655(2.7)$ & $221(1.1)$ & $7,639(12.1)$ \\
\hline \multicolumn{6}{|c|}{ Health care visits (d) } \\
\hline Mean & 58.1 & 55.1 & 87.6 & 126.9 & 25.6 \\
\hline Median & 28 & 37 & 55 & 83 & 13 \\
\hline \multicolumn{6}{|c|}{ Specific conditions ${ }^{*}$} \\
\hline \multicolumn{6}{|c|}{ Temporomandibular joint disorder } \\
\hline Yes & $2,631(2.3)$ & $224(2.4)$ & $647(2.6)$ & $779(4.0)$ & $981(1.6)$ \\
\hline No & $113,727(97.7)$ & $9,077(97.6)$ & $23,877(97.4)$ & $18,777(96.0)$ & $61,996(98.4)$ \\
\hline \multicolumn{6}{|c|}{ Metabolic disorders } \\
\hline Yes & $5,604(4.8)$ & $408(4.4)$ & $1,610(6.6)$ & $1,518(7.8)$ & 2,068 (3.3) \\
\hline No & $110,754(95.2)$ & $8,893(95.6)$ & $22,914(93.4)$ & $18,038(92.2)$ & $60,909(96.7)$ \\
\hline \multicolumn{6}{|l|}{ Autoimmune } \\
\hline Yes & $5,655(4.9)$ & $461(5.0)$ & $1,746(7.1)$ & $1,940(9.9)$ & $1,508(2.4)$ \\
\hline No & $110,703(95.1)$ & $8,840(95.0)$ & 22,778 (92.9) & $17,616(90.1)$ & $61,469(97.6)$ \\
\hline \multicolumn{6}{|c|}{ Blood vessel disorders } \\
\hline \multicolumn{6}{|l|}{ Hearing loss } \\
\hline Yes & $48,694(41.9)$ & $3,622(38.9)$ & 10,765 (43.9) & $8,742(44.7)$ & $25,565(40.6)$ \\
\hline No & $67,664(58.2)$ & $5,679(61.1)$ & $13,759(56.1)$ & $10,814(55.3)$ & $37,412(59.4)$ \\
\hline \multicolumn{6}{|c|}{ Cervical whiplash } \\
\hline Yes & $1,028(0.9)$ & $101(1.1)$ & $280(1.1)$ & $297(1.5)$ & $350(0.6)$ \\
\hline No & $115,330(99.1)$ & $9,200(98.9)$ & 24,244 (98.9) & $19,259(98.5)$ & $62,627(99.4)$ \\
\hline \multicolumn{6}{|c|}{ Cerebrovascular diseases } \\
\hline Yes & $934(0.8)$ & $59(0.6)$ & $302(1.2)$ & $297(1.5)$ & $276(0.4)$ \\
\hline No & $115,424(99.2)$ & $9,242(99.4)$ & $24,222(98.8)$ & $19,259(98.5)$ & $62,701(99.6)$ \\
\hline \multicolumn{6}{|l|}{ TBI } \\
\hline Yes & 26,632 (22.9) & 2,601 (28.0) & $8,517(34.7)$ & $7,636(39.1)$ & $7,878(12.5)$ \\
\hline No & $89,726(77.1)$ & $6,700(72.0)$ & 16,007 (65.3) & $11,920(60.9)$ & $55,098(87.5)$ \\
\hline
\end{tabular}

"Veterans can have multiple diagnoses. Thus, a Veteran is counted only once for any single condition, but can be counted in multiple conditions. Percentage values are shown in parentheses.

tinnitus/depression/anxiety, and 40.9 per 100,000 person-years tinnitus/depression subgroups, compared with 15.9 per 100,000 person-years in the tinnitus-only group.

\section{Likelihood of Mortality}

Mortality differences were further analyzed using a logistic regression model with no-tinnitus diagnosis as the reference group (Table 4). As evident in the Tinnitus Subgroups section of Table 4, there was no difference in the likelihood of suicide among Veterans diagnosed with tinnitus and depression and/or anxiety compared with Veterans with no tinnitus diagnosis. Yet, the likelihood of suicide among the tinnitus-only group [odds ratio $(\mathrm{OR})=0.4 ; 95 \% \mathrm{CI}=0.31-0.63$ ] was significantly lower than the no-tinnitus group. Further, the odds of dying (from any cause) were significantly lower among Veterans diagnosed with tinnitus, at half the rate as Veterans who were not diagnosed 


\begin{tabular}{|c|c|c|c|c|}
\hline & \multicolumn{4}{|c|}{ Death Rates per 100,000 Person Years } \\
\hline & \multicolumn{2}{|c|}{ All Cause } & \multicolumn{2}{|c|}{ Unintentional Health } \\
\hline & Death & Suicide & Injury & Related \\
\hline $\begin{array}{l}\text { Level 1: received health } \\
\text { care } 2002-2011\end{array}$ & 160.1 & 32.0 & 63.7 & 64.4 \\
\hline \multicolumn{5}{|l|}{ Level 2: any tinnitus } \\
\hline Yes & 131.6 & 26.9 & 60.0 & 44.7 \\
\hline No & 165.6 & 33.0 & 64.5 & 68.2 \\
\hline \multicolumn{5}{|l|}{ Level 3: tinnitus subgroups } \\
\hline Tinnitus with anxiety & 131.6 & 24.5 & 73.5 & 33.7 \\
\hline Tinnitus with depression & 165.9 & 40.9 & 73.5 & 51.4 \\
\hline $\begin{array}{l}\text { Tinnitus with depression } \\
\text { and anxiety }\end{array}$ & 179.2 & 39.5 & 100.1 & 39.5 \\
\hline Tinnitus only & 97.3 & 15.9 & 36.1 & 45.3 \\
\hline
\end{tabular}

with tinnitus $(\mathrm{OR}=0.5 ; 95 \% \mathrm{CI}=0.41-0.55)$. Specifically, Veterans diagnosed with tinnitus only were $60 \%$ less likely to die of suicide $(\mathrm{OR}=0.4 ; 95 \% \mathrm{CI}=0.31-0.60)$ and half as likely to die of either other unintentional injury $(\mathrm{OR}=0.5 ; 95 \% \mathrm{CI}=0.40$ $0.64)$ or health-related causes $(\mathrm{OR}=0.5 ; 95 \% \mathrm{CI}=0.38-0.59)$ compared with Veterans with no tinnitus diagnosis.

\section{Post hoc Analyses}

A post hoc analysis was performed to determine if tinnitus with co-occurring physical conditions exacerbated an individual's mental health and distress levels, resulting in increased risk of suicide. The likelihood of suicide was lower among individuals with tinnitus compared with those without tinnitus. However, the likelihood of death was higher among Veterans with a co-occurring physical condition compared with those with no co-occurring physical condition $(\mathrm{OR}=1.3 ; 95 \%$ $\mathrm{CI}=1.22-1.40)$, but the likelihood of suicide was lower $(\mathrm{OR}=0.8 ; 95 \% \mathrm{CI}=0.70-0.96)$. Regardless of the cause of death, the odds of dying were lower among Veterans diagnosed with tinnitus compared with those not diagnosed with this condition $(\mathrm{OR}=0.7 ; 95 \% \mathrm{CI}=0.61-0.73)$. Veterans who had an audiology or mental-health clinic stop were less likely to die of health-related causes $(\mathrm{OR}=0.9 ; 95 \% \mathrm{CI}=0.80-0.99)$ and more likely to die due to suicide $(\mathrm{OR}=2.3 ; 95 \% \mathrm{CI}=1.95-2.71)$ or unintentional injury $(\mathrm{OR}=1.5 ; 95 \% \mathrm{CI}=1.38-1.72)$ compared with Veterans with no audiology or mental-health clinic stop.

A second post hoc analysis was performed to test for interactions between tinnitus and depression and tinnitus and attempted self-harm with the outcome of suicide. No interactions were found, leading to the deduction that Veterans diagnosed with tinnitus have a decreased likelihood of death caused by suicide compared with Veterans with no tinnitus diagnosis.

\section{DISCUSSION}

The two goals of this study were to investigate whether there was a significant association between tinnitus diagnosis and suicide and whether depression and anxiety strengthened that association. The likelihood of suicide among the tinnitus group was significantly lower than the no-tinnitus group; thus, the first hypothesis that a diagnosis of tinnitus was positively associated with suicide was not supported. Further, when examining the four tinnitus subgroups, the likelihood of suicide was significantly lower in the tinnitus-only group than the no-tinnitus group. There was a higher percentage of death due to health-related or unintentional injury causes than by suicide among Veterans receiving VA health care. The same pattern was observed among Veterans diagnosed with tinnitus: the proportion of unintentional injury and health-related deaths was greater than the proportion of suicide deaths.

Having depression marginally increased the occurrence of suicide among individuals with tinnitus; however, the odds differences were not statistically significant in predicting suicide. This did not support the second hypothesis that depression and anxiety strengthened the association between tinnitus diagnosis and suicide.

Co-occurring physical conditions were also examined to determine if they explained the lower risk of suicide observed among Veterans diagnosed with tinnitus. The contribution of co-occurring conditions to mortality was examined based on the supposition that physical health complications other than tinnitus might dominate Veterans' health care encounters and individuals' psychological health concerns. Almost two-thirds of those with a tinnitus diagnosis had at least one co-occurring physical condition, which might be indicative of a poorer overall health status. A post hoc analysis examined the possibility that a co-occurring physical condition might exacerbate individuals' mental health and distress levels, which might increase the risk of suicide. Findings indicated that having a co-occurring physical condition decreased the odds of dying from unintentional injury or suicide, but increased the likelihood of dying from health-related causes. A second post hoc analysis tested for interactions between (1) tinnitus and depression and (2) tinnitus and attempted self-harm with the outcome of suicide and found no interactions.

\section{Psychological States and Tinnitus}

Clinical and anecdotal reports were that having tinnitus, especially at severe and intense levels, may increase the risk of suicide. Checking the veracity of this association is what compelled the present research team to conduct this investigation. The results of this investigation indicated that, after controlling for several factors, the risk of suicide was lower in the group diagnosed with tinnitus compared with the group without a tinnitus diagnosis. This indicated that having a diagnosis of tinnitus, a chronic impairment, was not associated with an increased likelihood of suicide in this sample. This finding mirrors case-control study by Dobscha et al. (2014) that focused on suicide risk factors among Veterans receiving health care from the VA: they found that having a VA serviceconnected disability was associated with a decreased risk of suicide.

Researchers have acknowledged that the association between tinnitus and suicide is a complex one (Jacobson \& McCaslin, 2001; Langguth et al., 2011; Lewis, 2002; Nondahl et al., 2011), with some stating that they could not assume a causal relationship (Jacobson \& McCaslin, 2001; Pridmore et al., 2012). The lack of a positive association between a tinnitus diagnosis and suicide may seem counterintuitive, but it can be explained by some concepts from the field of rehabilitation psychology. It is possible that individuals who have tinnitus have learned to cope with tinnitus-related distress (Martz \& Henry, 2016), and these 


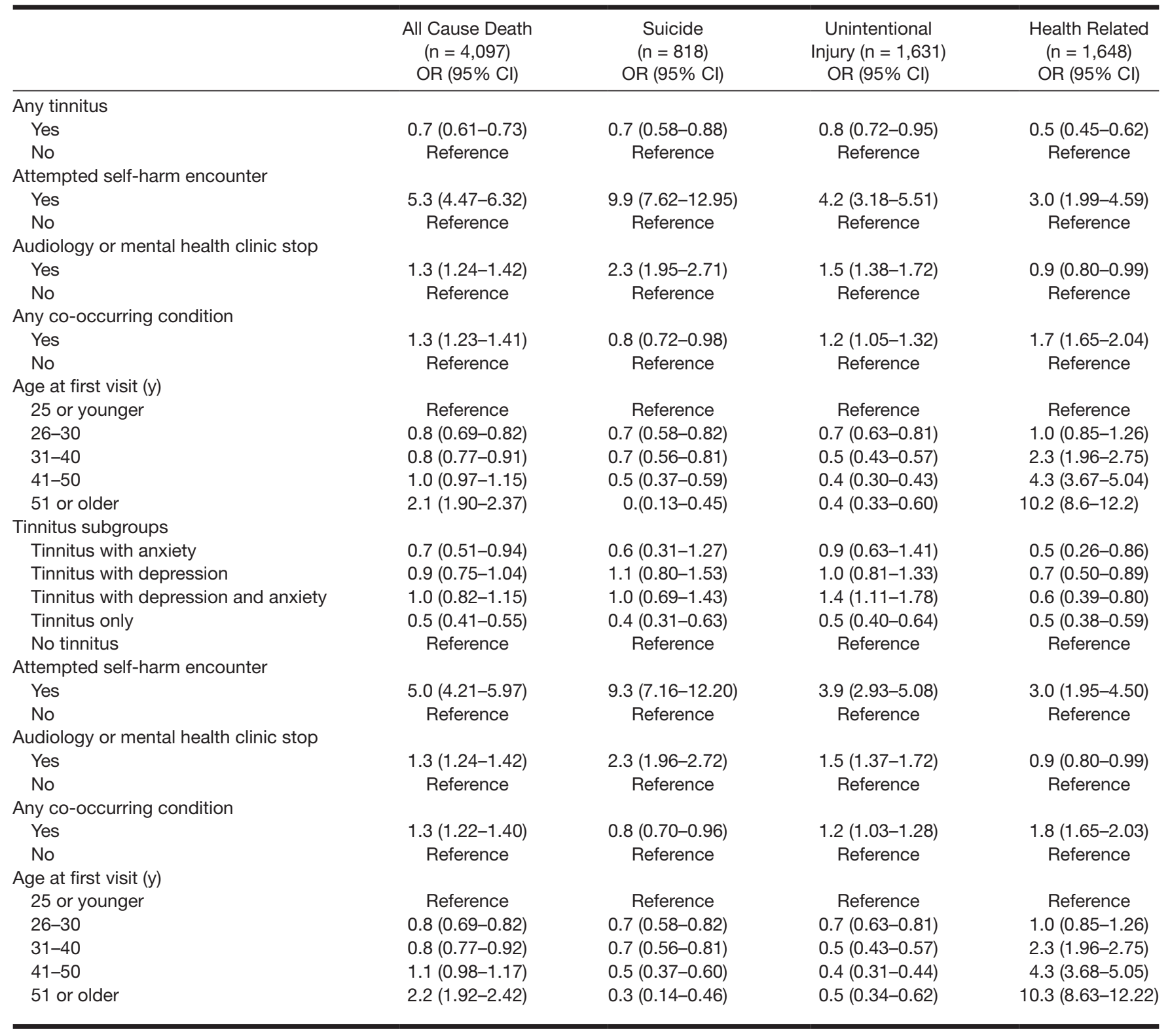

OR, odds ratio; Reference, the reference group of the odds ratio.

coping skills may generalize to managing the stress in other aspects of individuals' lives. Given that coping strategies are conscious efforts to manage stress (Lazarus \& Folkman, 1984; Martz \& Livneh, 2007; Zeidner \& Endler, 1996), it is possible that as individuals learn how to cope with tinnitus, their ability to cope with other stress in their lives increases, decreasing the likelihood of suicide. Indeed, effective coping and problem-solving skills have been associated with a decreased risk of suicide (Turecki \& Brent, 2016).

Several articles suggested that tinnitus annoyance (i.e., discomfort) was not correlated to neurobiological indicators, but rather correlated with psychological reactions, such as depression and anxiety (Granjeiro et al., 2013; Sampaio et al., 2013). These ideas suggest that it may not be the fact of having tinnitus that causes distress, but rather the cognitive and emotional processes and responses that may create the tinnitus-related annoyance or disturbance. The results of the present study concur with these ideas: tinnitus alone is not a significant risk factor for suicide.

The World Health Organization (2014) notes that the risk of suicide is directly or indirectly influenced by a combination of factors from several life domains (e.g., variables related to health systems, society, community, individual, or relationships), suggesting that a multidimensional approach should be used when researching suicide. In the present study, the statistical analyses performed tested the magnitude of the association between a diagnosis of tinnitus and suicide; it did not test causality. The statistical results did not support the hypothesis that there was a positive relationship between a diagnosis of tinnitus and suicide. The clinical assumption inherent in the concept that "tinnitus may lead to suicide" can also be challenged by a perspective offered for decades by rehabilitation psychologists: that the strength of negative psychological states (e.g., a clinical 
diagnosis of depression or anxiety) cannot be predicted solely by the onset of a chronic impairment or disability. In the study by Shontz (1977), six principles on disability and psychological adjustment, he emphasized that:

Psychological reactions to the onset or imposition of physical disability are not uniformly disturbing or distressing and do not necessarily result in maladjustment... Knowledge of the type or degree of a person's physical disability provides virtually no information about that person's personality (p. 208, emphasis added).

Empirical studies in various branches of psychology have affirmed Shontz's principles. For example, Dijkers (1997) metaanalysis of 22 studies indicated a weak association between impairment and quality of life among individuals with spinal cord injuries. Geyh et al. (2013) found that the type of spinal cord injury was not a significant predictor of quality of life. Schweininger et al. (2015) utilized structural equation modeling to examine temporal relationships between mental health and disability (i.e., functioning) after hospitalization for physical injury and found that injury severity (i.e., the anatomical impact) was not a significant predictor of disability, anxiety, or depression in the hospital, nor at 3- or 12-months post injury. These studies challenge the clinical assumption that having a chronic impairment like tinnitus may be a risk factor for suicide, a view that prompted the present study. In summary, these rehabilitation psychology perspectives suggest that the onset of tinnitus would not necessarily predict that individuals would have psychological reactions that reached clinical thresholds (e.g., a diagnosis of clinical depression or anxiety), or that an individual would commit suicide due to the onset of tinnitus. Again, this emphasizes that the physical indicators or anatomical impact of a chronic health condition is not indicative of the individual's mental health status. Other personality factors, including coping skills, may provide more information about how an individual responds to a chronic impairment like tinnitus.

\section{Clinical Implications}

In this study, $15 \%$ of Veterans were diagnosed with tinnitus, which suggests that healthcare providers for Veterans should be attentive to the possibility that their health care recipient may have tinnitus. Despite the findings that a tinnitus diagnosis was not directly associated with suicide nor was that association strengthened by having a diagnosis of depression or anxiety, it is important for clinicians to assess Veterans for symptoms of depression or anxiety because there is a high rate of cooccurrence in this sample: $46 \%$ of this sample had tinnitus with depression, anxiety, or both. If depression and anxiety co-occurs with tinnitus, then individuals should receive clinical attention to help decrease their symptomology and increase their quality of life. Further, even though the present research did not find that tinnitus was a risk factor for suicide when examining group data, some individuals who have tinnitus may be suicidal and may need clinical treatment. Jacobson and McCaslin (2001) proposed that if individuals have both significantly distressing tinnitus and symptoms that suggest clinical depression, then two types of professional support should be proffered: psychological/ psychiatric management and non-medical management of tinnitus. This advice can also be utilized in the context of increased anxiety and tinnitus.
Tunkel et al. (2014) reported that often individuals with tinnitus are told by clinicians that "little or nothing can be done to help them" (p. S18); this message may increase individuals' depressive reactions (e.g., the symptoms of helplessness and hopelessness), at least until they learn about the management techniques that are available for tinnitus. These kinds of responses from clinicians may reinforce feelings of frustration surrounding a tinnitus diagnosis and may even deter the individual from seeking further care for their tinnitus. This may be especially detrimental if the belief that "nothing can be done" leads to an individual not seeking treatment when symptoms of depression arise.

Given the findings that self-harm attempts were linked with higher rates of mortality and suicide (while not considering tinnitus), it may also be important for clinicians to assess individuals with tinnitus for self-harm tendencies, not just for symptoms of depression. Self-harm attempts may serve as an early warning sign for future episodes of depression and an increased risk for suicide (Klonsky et al., 2013; Victor \& Klonsky, 2014; Zahl \& Hawton, 2004).

Previous studies have shown the complex relationship between tinnitus and depression and suicide (Jacobson \& McCaslin, 2001; Langguth et al., 2011; Lewis, 2002; Nondahl et al., 2011). A multidisciplinary approach, which utilizes several intervention modalities tailored to the individual, may provide the best possible treatment outcomes. The results from this study underscore the importance of a multidisciplinary approach when treating individuals with tinnitus because it was found that individuals with a diagnosis of a co-occurring physical condition (while not considering a tinnitus diagnosis) are less likely to die by suicide. This suggests that health care professionals helping with physical conditions, and not just psychologists or psychiatrists, may play a role in decreasing individuals' risk for suicide.

\section{Limitations}

This analysis was limited in several respects. First, case identification was based on ICD-9-CM codes, increasing the potential for misclassification if an ICD-9-CM code did not represent a confirmed diagnosis. The case definition for tinnitus was based on VA coding guidelines, which indicate that a diagnostic code should only be used for a final diagnosis, not evaluation. Specifically, the guidelines state "do not code diagnoses documented as 'probable,' 'suspected,' 'questionable,' 'rule out,' or a 'working diagnosis"'; the coder is directed to code the condition "to the highest degree of certainty" using signs and symptoms that are documented (Department of Veterans Affairs, 2006). Case validation was not possible due to the reliance on administrative data from VA medical records. The training and guidance provided to coding professionals would reduce the likelihood of misclassification; thus, it was determined that the diagnostic codes would be used to define cases. Records for health care received outside of the VA were not obtained. Rosen et al. (2008) noted some of the limitations of using administrative data in research included "diagnostic uncertainty, incomplete data, and non-standardized data collection" (p. 295), which could have resulted in an underestimation of diagnoses, such as tinnitus, anxiety, depression, or suicide.

Most types of tinnitus are subjective, which means that a health care professional has to rely on self-reported symptoms rather than objective measurements to make a diagnosis (Hobson et al., 2012). In the present study, the subjective 
severity level of tinnitus could not be assessed due to the nature of the data. While the research team discussed utilizing Veterans benefits' data as a proxy for severity, it was agreed that it would not accurately represent the severity of tinnitus. The lack of a valid measure of severity may be a limitation of this study, but decades of rehabilitation research indicates that information about the anatomical impact or severity of disability typically is not a significant predictor of psychological reactions to chronic impairment and disability; thus, information about the severity of tinnitus would likely not have influenced the results of this study.

As previously noted, no causation should be implied by this study; the analysis tested for the magnitude of association between a tinnitus diagnosis and suicide, not the causation. Also, the data of this study were drawn from a specific group of Veterans in the United States; hence, the results cannot be generalized outside of the population studied in this research. Because no psychological assessments on coping or resilience or instruments that assessed help-seeking behavior were available in the dataset, assumptions cannot be made about the psychological, medical, and social resources of Veterans with tinnitus. Veterans who have displayed help-seeking behaviors by utilizing the services at the VA may have psychological qualities that are different than the Veterans who do not seek help at the VA; this kind of data were unavailable to the researchers. However, such a comparison would be useful in future research. The conclusions drawn from this study should be viewed as tentative. More research is required to confirm the validity of the results.

\section{CONCLUSION}

The purpose of this article was to explore associations between tinnitus, depression, anxiety, and suicide by means of a retrospective data analysis. While neither of the hypotheses was supported, the results help to answer the question about whether individuals with a diagnosis of tinnitus were at increased risk of suicide. The lack of association between tinnitus and suicide could be explained by theories from rehabilitation psychology, which propound that the severity of psychological reactions to the onset of chronic impairment or disability cannot be predicted by any physical indicators or anatomical impact (e.g., severity) of an impairment or disability. This perspective was confirmed by this study: having a diagnosis of tinnitus does not significantly increase the risk for suicide among Veterans receiving health care in the VA system.

Future research can seek to replicate these results in other populations. Future research also can focus on psychological interventions that target mental-health issues (e.g., anxiety or depression) experienced by individuals with tinnitus, in order to help increase individuals' overall quality of life.

\section{ACKNOWLEDGEMENTS}

This material is the result of work supported with resources and the use of facilities at the VA Portland Healthcare System and the support of the VHA Office of Patient Care Services, Post Deployment Health Services. The authors acknowledge the help that Dr. Robert Bossarte provided to the first two authors as they were starting this research study.

Disclaimer: The views expressed in this article are those of the authors and do not necessarily reflect the position or policy of the Department of Veterans Affairs or the United States government.

All authors contributed equally to this work. E.M. and C.J. conducted the literature review, and E.M. wrote most of the introduction and discussion sections. D.D. and C.W. ran the data analyses and wrote most of the Methods section, including the results, and created the tables. A.S. reviewed and discussed the data analyses with D.D. and C.W. All authors not only discussed the results and their implications but also edited the article throughout its evolution.

There are no conflicts of interest to report.

Address for correspondence: Erin Martz, VA Portland Healthcare System, Bldg. 6, Room 114A, 3710 SW U.S. Veterans Hospital Rd, Portland, OR 97239. E-mail: rehabilityoregon@gmail.com

Received June 27, 2017; accepted January 23, 2018.

\section{REFERENCES}

Bartels, H., Middel, B. L., van der Laan, B. F., et al. (2008). The additive effect of co-occurring anxiety and depression on health status, quality of life and coping strategies in help-seeking tinnitus sufferers. Ear Hear, 29, 947-956.

Bhatt, J. M., Bhattacharyya, N., Lin, H. W. (2017). Relationships between tinnitus and the prevalence of anxiety and depression. Laryngoscope, $127,466-469$.

Cave, K. M., Cornish, E. M., Chandler, D. W. (2007). Blast injury of the ear: clinical update from the global war on terror. Mil Med, 172, 726-730.

Chesney, E., Goodwin, G. M., Fazel, S. (2014). Risks of all-cause and suicide mortality in mental disorders: a meta-review. World Psychiatry, 13, $153-160$.

Dijkers, M. (1997). Quality of life after spinal cord injury: a meta analysis of the effects of disablement components. Spinal Cord, 35, 829-840.

Department of Veterans Affairs. (2006). Handbook for Coding Guidelines version 6.0. Retrieved November 9, 2016 from http://opf-labs.org/format-corpus/govdocs1-error-pdfs/error_set_1/316267.pdf.

Dobscha, S. K., Denneson, L. M., Kovas, A. E., et al. (2014). Correlates of suicide among veterans treated in primary care: case-control study of a nationally representative sample. J Gen Intern Med, 29(Suppl 4), 853-860.

Erlandsson, S. I., \& Hallberg, L. R. (2000). Prediction of quality of life in patients with tinnitus. BrJ Audiol, 34, 11-20.

Fausti, S. A., Wilmington, D. J., Gallun, F. J., et al. (2009). Auditory and vestibular dysfunction associated with blast-related traumatic brain injury. J Rehabil Res Dev, 46, 797-810.

Gaziano, J. M., Concato, J., Brophy, M., et al. (2016). Million Veteran Program: a mega-biobank to study genetic influences on health and disease. $J$ Clin Epidemiol, 70, 214-223.

Geyh, S., Ballert, C., Sinnott, A., et al. (2013). Quality of life after spinal cord injury: a comparison across six countries. Spinal Cord, 51, 322-326.

Granjeiro, R. C., Kehrle, H. M., de Oliveira, T. S., et al. (2013). Is the degree of discomfort caused by tinnitus in normal-hearing individuals correlated with psychiatric disorders? Otolaryngol Head Neck Surg, $148,658-663$.

Harrop-Griffiths, J., Katon, W., Dobie, R., et al. (1987). Chronic tinnitus: association with psychiatric diagnoses. J Psychosom Res, 31, 613-621.

Hawton, K., Casañas I Comabella, C., Haw, C., et al. (2013). Risk factors for suicide in individuals with depression: a systematic review. $J$ Affect Disord, 147, 17-28.

Hoare, D. J., Edmondson-Jones, M., Sereda, M., et al. (2014). Amplification with hearing aids for patients with tinnitus and co-existing hearing loss. Cochrane Database Syst Rev, 1, CD010151.

Hobson, J., Chisholm, E., El Refaie, A. (2012). Sound therapy (masking) in the management of tinnitus in adults. Cochrane Database Syst Rev, 11, CD006371.

Hoffmire, C. A., Kemp, J. E., Bossarte, R. M. (2015). Changes in suicide mortality for veterans and nonveterans by gender and history of VHA service use, 2000-2010. Psychiatr Serv, 66, 959-965.

Jacobson, G. P., \& McCaslin, D. L. (2001). A search for evidence of a direct relationship between tinnitus and suicide. J Am Acad Audiol, 12, 493-496.

Joo, Y. H., Han, K. D., Park, K. H. (2015). Association of hearing loss and tinnitus with health-related quality of life: The Korea National Health and Nutrition Examination Survey. PLoS One, 10, e0131247.

Kang, H. K., Bullman, T. A., Smolenski, D. J., et al. (2015). Suicide risk among 1.3 million veterans who were on active duty during the Iraq and Afghanistan wars. Ann Epidemiol, 25, 96-100.

Kanwar, A., Malik, S., Prokop, L. J., et al. (2013). The association between anxiety disorders and suicidal behaviors: a systematic review and metaanalysis. Depress Anxiety, 30, 917-929. 
Kemp, J., \& Bossarte, R. (2012). Suicide data report, 2012. Department of Veterans Affairs Mental Health Services Suicide Prevention Program. Washington, DC: Office of Public and Intergovernmental Affairs.

Klonsky, E. D., May, A. M., Glenn, C. R. (2013). The relationship between nonsuicidal self-injury and attempted suicide: converging evidence from four samples. J Abnorm Psychol, 122, 231-237.

KochKin, S., Tyler, R., \& Born, J. (2011). MarkeTrak VIII: the prevalence of tinnitus in the United States and the self-reported efficacy of various treatments. Hear Rev, 18, 10-27.

Krog, N. H., Engdahl, B., Tambs, K. (2010). The association between tinnitus and mental health in a general population sample: results from the HUNT Study. J Psychosom Res, 69, 289-298.

Langguth, B., Landgrebe, M., Kleinjung, T., et al. (2011). Tinnitus and depression. World J Biol Psychiatry, 12, 489-500.

Lazarus, R., \& Folkman, S. (1984). Stress, Coping, and Adaptation. New York, NY: Springer.

Lewis, J. E. (2002). Tinnitus and suicide. J Am Acad Audiol, 13, 339; author reply 339-339; author reply 341 .

Lewis, J., Stephens, D., Huws, D. (1992). Suicide in tinnitus sufferers. J Audiologic Med, 1, 30-37.

Lewis, J. E., Stephens, S. D., McKenna, L. (1994). Tinnitus and suicide. Clin Otolaryngol Allied Sci, 19, 50-54.

Marciano, E., Carrabba, L., Giannini, P., et al. (2003). Psychiatric comorbidity in a population of outpatients affected by tinnitus: comorbilidad psiquiátrica en una población de pacientes de consulta externa afectados por tinnitus. Int $J$ Audiol, 42, 4-9.

Martz, E., \& Henry, J. A. (2016). Coping with tinnitus. J Rehabil Res Dev, 53, 729-742.

Martz, E., \& Livneh, H. (2007). Coping with Chronic Illness and Disability. New York, NY: Springer.

McCormack, A., Edmondson-Jones, M., Fortnum, H., et al. (2015). Investigating the association between tinnitus severity and symptoms of depression and anxiety, while controlling for neuroticism, in a large middle-aged UK population. Int J Audiol, 54, 599-604.

Myers, P. J., Wilmington, D. J., Gallun, F. J., et al. (2009). Hearing impairment and traumatic brain injury among soldiers: special considerations for the audiologist. In Seminars in Hearing (Vol. 30, No. 01, pp. 005-027). Stuttgart, Germany: Thieme Medical Publishers.

Nondahl, D. M., Cruickshanks, K. J., Huang, G. H., et al. (2011). Tinnitus and its risk factors in the Beaver Dam offspring study. Int JAudiol, 50, 313-320.

Pridmore, S., Walter, G., Friedland, P. (2012). Tinnitus and suicide: recent cases on the public record give cause for reconsideration. Otolaryngol Head Neck Surg, 147, 193-195.

Reger, M. A., Smolenski, D. J., Skopp, N. A., et al. (2015). Risk of suicide among US military service members following Operation Enduring Freedom or Operation Iraqi Freedom Deployment and Separation From the US Military. JAMA Psychiatry, 72, 561-569.
Rosen, C. S., Kuhn, E., Greenbaum, M. A., et al. (2008). Substance abuserelated mortality among middle-aged male VA psychiatric patients. $P s y-$ chiatr Serv, 59, 290-296.

Sampaio, A. L., Kehrle, H. M., Oliveira, T. S., et al. (2013). Does tinnitus annoyance correlate to auditory brainsteam responses? Otolaryngol Head Neck Surg, 149 (2 Suppl), P222-P222.

Schweininger, S., Forbes, D., Creamer, M., et al. (2015). The temporal relationship between mental health and disability after injury. Depress Anxiety, 32, 64-71.

Seo, J. H., Kang, J. M., Hwang, S. H., et al. (2016). Relationship between tinnitus and suicidal behaviour in Korean men and women: a cross-sectional study. Clin Otolaryngol, 41, 222-227.

Shontz, F. C. (1977). Six principles relating disability and psychological adjustment. Rehabil Psychol, 24, 207-210.

Sullivan, M. D., Katon, W., Dobie, R., et al. (1988). Disabling tinnitus. Association with affective disorder. Gen Hosp Psychiatry, 10, 285-291.

Sullivan, M., Katon, W., Russo, J., et al. (1993). A randomized trial of nortriptyline for severe chronic tinnitus. Effects on depression, disability, and tinnitus symptoms. Arch Intern Med, 153, 2251-2259.

Tunkel, D. E., Bauer, C. A., Sun, G. H., et al. (2014). Clinical practice guideline: tinnitus. Otolaryngol Head Neck Surg, 151(2 Suppl), S1-S40.

Turecki, G., \& Brent, D. A. (2016). Suicide and suicidal behaviour. Lancet, $387,1227-1239$.

Veterans Affairs, U. S. D. O. (2016). Suicide Among Veterans and Other Americans. Washington, D.C.: Department of Veterans Affairs.

Veterans Benefits Administration. (2016). VBA annual report Veterans Benefits Administration, Department of Veterans Affairs. Retrieved on September 13, 2017 from https://www.benefits.va.gov/REPORTS/abr/ ABR-Compensation-FY16-0613017.pdf.

Victor, S. E., \& Klonsky, E. D. (2014). Correlates of suicide attempts among self-injurers: a meta-analysis. Clin Psychol Rev, 34, 282-297.

World Health Organization (2014). Preventing Suicide: A Global Imperative. Geneva, Switzerland: World Health Organization.

Yankaskas, K. (2013). Prelude: noise-induced tinnitus and hearing loss in the military. Hear Res, 295, 3-8.

Yurgil, K. A., Clifford, R. E., Risbrough, V. B., et al.; MRS Team. (2016). Prospective associations between traumatic brain injury and postdeployment tinnitus in active-duty marines. J Head Trauma Rehabil, 31, 30-39.

Zahl, D. L., \& Hawton, K. (2004). Repetition of deliberate self-harm and subsequent suicide risk: long-term follow-up study of 11,583 patients. $B r$ $J$ Psychiatry, 185, 70-75.

Zeidner, M., \& Endler, N. S. (1996). Handbook of Coping: Theory, Research, Applications (Vol. 195). New York, NY: John Wiley \& Sons.

Zivin, K., Yosef, M., Miller, E. M., et al. (2015). Associations between depression and all-cause and cause-specific risk of death: a retrospective cohort study in the Veterans Health Administration. J Psychosom Res, 78, $324-331$. 